\title{
Charge Carrier Photogeneration and Transport Properties of a Novel Low-Bandgap Conjugated Polymer for Organic Photovoltaics
}

\author{
Cesare Soci ${ }^{*}$, In-Wook Hwang ${ }^{a}$, Cuiying Yang ${ }^{\mathrm{a}}$, Daniel Moses ${ }^{\mathrm{a}}$, Zhengguo Zhu ${ }^{\mathrm{b}}$, David Waller ${ }^{\mathrm{b}}$, \\ Russel Gaudiana ${ }^{\mathrm{b}}$, Christoph J. Brabec ${ }^{\mathrm{c}}$, and Alan J. Heeger ${ }^{\mathrm{a}}$ \\ ${ }^{a}$ Center for Polymers and Organic Solids, Univ. of California Santa Barbara, Santa Barbara, CA \\ 93106-5090, USA; \\ ${ }^{\mathrm{b}}$ Konarka Technologies, Inc., 116 John Street, Lowell, MA 01852, USA; \\ ${ }^{c}$ Konarka Austria Forschungs und Entwicklungs GmbH, Altenbergerstrasse 69, 4040 Linz, Austria
}

\begin{abstract}
The overall power conversion efficiency of organic solar cells depends on many factors, some of which such as photon absorption, charge carrier photogeneration, separation and transport are intrinsic properties of the active material. The use of low-bandgap conjugated polymers in polymer/fullerene bulk heterojunctions improves the spectral overlap between the polymer absorption and the solar irradiance spectrum, and is therefore a promising route toward increased light harvesting and higher power conversion efficiency of polymer photovoltaics. We present our studies on the optical and charge transport properties of a novel low-bandgap conjugated polymer, poly[2,6-(4,4-bis-(2-ethylhexyl)- $4 H$ cyclopenta[2,1-b;3,4-b']dithiophene)-alt-4,7-(2,1,3-benzothiadiazole)], PCPDTBT, with an optical energy gap of $\mathrm{E}_{\mathrm{g}}=1.46 \mathrm{eV}$. The combination of steady-state and transient photoconductivity with photoinduced absorption measurements has allowed us to investigate the charge carrier photogeneration and charge transport mechanisms in pristine PCPDTBT and PCPDTBT:PCBM interpenetrating networks, and to compare them to the P3HT and P3HT:PCBM model systems. The picture of the photophysics of PCPDTBT:PCBM emerging from these studies is very similar to that of P3HT:PCBM blends. We discuss the potential of PCPDTBT as a new material for high efficiency polymer solar cells.
\end{abstract}

Keywords: Low-bandgap conjugated polymers, organic photovoltaics, photoconductivity, photoinduced absorption

\section{INTRODUCTION}

The efficient conversion and utilization of solar energy is widely recognized as a possible solution to the global energy crisis and the greenhouse problem. The challenge in converting sunlight to electricity via photovoltaic solar cells is to considerably reduce the cost per watt of delivered solar electricity to be able to compete with currently available energy sources. ${ }^{1}$ Conjugated polymers are amongst the most promising materials to replace silicon and inorganic semiconductors in the future "third generation" thin-film photovoltaics, ${ }^{2}$ due to their low cost and ease of processing. So far, the major limiting factor to the commercialization of polymer photovoltaics has been their low power conversion efficiency. The best results have been reported from bulk heterojunctions (BHJs) comprising of poly(3-hexylthiophene), P3HT (Fig. 1a), and the [6,6]-phenyl- $\mathrm{C}_{61}$ butyric acid methyl ester fullerene derivative, PCBM (Fig. 1b), which yielded power conversion efficiencies approaching 5\%. ${ }^{3,4}$ In such BHJ structures, ultrafast photoinduced charge transfer occurs at the interface between the polymer (donor) and the fullerene (acceptor) and results in charge separation and the generation of positive and negative charge carriers. Provided that the back transfer rate and geminate recombination are sufficiently slow, the mobile positive and negative carriers generated by the ultrafast electron transfer reaction can be transported and collected at the opposite electrodes (typically indium-tin oxide and a lower work-function metal such as $\mathrm{Al})$. The carries collection is driven by the internal field created by the different work-functions of the two electrodes in the metal/insulator/metal configuration (electrons are collected at the lower work function electrode and holes at the higher work function electrode).

The ultimate efficiency of polymer/fullerene BHJ solar cells depends on many factors, including light harvesting, charge carrier photogeneration (charge separation) and charge transport. ${ }^{6,7,8}$ The conjugated polymers that have shown the highest power conversion efficiencies are the poly(p-phenylene vinylene)s (PPVs) and the polythiophenes (PTs), ${ }^{9,10}$

Organic Photovoltaics VII, edited by Zakya H. Kafafi, Paul A. Lane,

Proc. of SPIE Vol. 6334, 63340D, (2006) $\cdot 0277-786$ X/06/\$15 $\cdot$ doi: 10.1117/12.680781

Proc. of SPIE Vol. 6334 63340D-1 
with absorption spectra that onset at $\sim 2 \mathrm{eV}$ and $\sim 1.8 \mathrm{eV}$, respectively. Despite their good charge carrier photogeneration and charge transport properties, the absorption spectra of these polymers overlap poorly with the solar emission spectrum, which peaks at $\sim 2.5 \mathrm{eV}$ but extends deep into the infrared. Thus, new low-bandgap materials to efficiently absorb sunlight over the full spectrum of wavelengths in solar radiation have been recently researched to achieve higher photovoltaic efficiency. ${ }^{10,11}$

One successful approach to low bandgap conjugated polymers is the synthesis of copolymers comprising alternating electron-rich and electron-deficient units, such as poly(thienylpyrrolethienyl-alt-benzothiadiazole), PTPTB ( $\mathrm{E}_{\mathrm{q}} \sim 1.6$ $\mathrm{eV}),{ }^{12,13,14,15}$ and poly(fluorene-alt-dithienylbenzothiadiazole), PFDTBT $\left(\mathrm{E}_{\mathrm{g}}=1.9 \mathrm{eV}\right) .{ }^{16,17}$ Although composites of these copolymers with PCBM have shown improved photon harvesting and efficient photogeneration of charged separated states, typical solar energy conversion efficiencies achieved in these systems are limited to $\sim 1 \%$ for PTPTB:PCBM ${ }^{14}$ and $\sim 2.2 \%$ for PFDTBT:PCBM. ${ }^{16}$

(a)

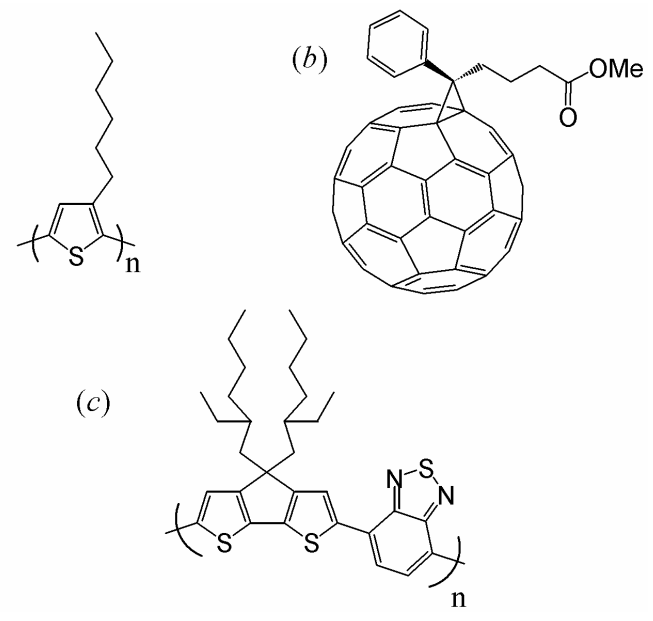

Fig. 1. Molecular structures of: (a) P3HT, poly-(3-hexylthiophene); (b) PCBM, [6,6]-phenyl- $\mathrm{C}_{61}$ butyric acid methyl ester; and (c) PCPDTBT, poly[2,6-(4,4-bis-(2-ethylhexyl)-4H-cyclopenta[2,1-b;3,4-b']dithiophene)-alt-4,7-(2,1,3benzothiadiazole). The molecular weight of the PCPDTBT used was approximately $30000 \mathrm{gmol}^{-1}$.

Here we describe the studies of a novel low band-gap copolymer, cyclopentadithiophene-alt-benzothiadiazole (PCPDTBT), made of alternating electron-rich and electron-deficient units (the molecular structure of PCPDTBT is reported in Fig. 1c). The optical, morphological, charge carrier photogeneration and transport properties of PCPDTBT, PCPDTBT:PCBM and PCPDTBT:P3HT:PCBM composites have been investigated through measurements of absorbance, photoluminescence (PL), transmission electron microscopy (TEM), photoinduced absorption (PIA) and photoconductivity (PC), and compared to those of the P3HT and P3HT:PCBM model systems. The picture of the photophysics of PCPDTBT:PCBM bulk heterojunctions emerging from these studies is very similar to that of P3HT:PCBM blends: PCPDTBT:PCBM shows low-energy absorption edge $\left(E_{\mathrm{g}} \sim 1.46 \mathrm{eV}\right)$, ultrafast photoinduced electron transfer reaction with quantum efficiency close to unity and good photoconductive properties, which make it suitable for the realization of high efficiency solar cells.

\section{EXPERIMENTAL DETAILS}

PCPDTBT and PCBM were provided by Konarka Technology, Inc. Details on the synthesis of PCPDTBT will be presented in a separate publication. ${ }^{18}$ Samples were prepared in a nitrogen glove-box with oxygen count of $\sim 1.5 \mathrm{ppm}$. P3HT and P3HT:PCBM blends were dissolved in chlorobenzene (1\% wt), while PCPDTBT and PCPDTBT:PCBM blends were dissolved in ortho-dichlorobenzene $(1 \% \mathrm{wt})$. Thin polymer films were obtained by spin-casting the polymer solutions on quartz or alumina substrates at $1000 \mathrm{rpm}$, while thick $(\sim 1 \mu \mathrm{m})$ films were formed by drop-casting the solutions on quartz or $\mathrm{KBr}$ substrates. The samples were thermally annealed in a vacuum chamber at $150{ }^{\circ} \mathrm{C}$ for 30 minutes. 
The absorption spectra were recorded by an automatic, dual beam spectrometer, while the photoluminescence spectra by a fluorimeter equipped with a Xenon lamp. The samples were excited by monochromatic light $(\lambda=750 \mathrm{~nm})$ at an incidence angle of $\sim 45^{\circ}$ and the emitted photoluminescence was collected at $\sim 45^{\circ}$, dispersed by a monochromator, and recorded by a liquid nitrogen cooled Ge detector.

For photoconductivity measurements, polymer films were deposited on alumina substrates and metallic $(\mathrm{Au})$ electrodes were thermally evaporated onto the film surface. The contacts formed a fast micro-strip transmission line (Auston switch $)^{19}$ with a gap between the electrodes of $100 \mu \mathrm{m}$, which allowed both steady-state and transient measurements with temporal resolution as high as $100 \mathrm{ps}$. Photocurrent transients were generated by pulsed laser photoexcitation under applied electric field $\left(\mathrm{F} \sim 1 \times 10^{4} \mathrm{~V} / \mathrm{cm}\right)$ and the photocurrent waveforms were recorded by a Boxcar integrator. The laser pulses (100 fs duration, $1 \mathrm{KHz}$ repetition rate) were produced by a Ti:sapphire system, where an optical parametric amplifier (OPA) was used to tune the excitation wavelength at the absorption peak of the polymers. Steady-state photoconductivity measurements were carried out with the conventional lock-in technique; the light source was a Xenon lamp and the light modulation frequency was $v=166 \mathrm{~Hz}$. The data were normalized by the measuring system response obtained after each run with a calibrated Si photodiode. All measurements were performed in vacuum $\left(\mathrm{P}<10^{-4}\right.$ Torr $)$.

Steady-state PIA measurements in the far-infrared were obtained at low temperature $(90 \mathrm{~K})$ by transmittance $(\mathrm{T})$ measurements in an automatic FTIR spectrometer equipped with a cold finger cryostat. Thick films deposited on $\mathrm{KBr}$ substrates were utilized. The excitation source was a CW Ar+ laser. The average of multiple (200) transmission spectra recorded alternately in the dark $\left(\mathrm{T}_{\text {dark }}\right)$ and under illumination $\left(\mathrm{T}_{\text {light }}\right)$ was used to obtain the photoinduced absorption as $-\left(\mathrm{T}_{\text {light }}-\mathrm{T}_{\text {dark }}\right) / \mathrm{T}_{\text {dark }}$.

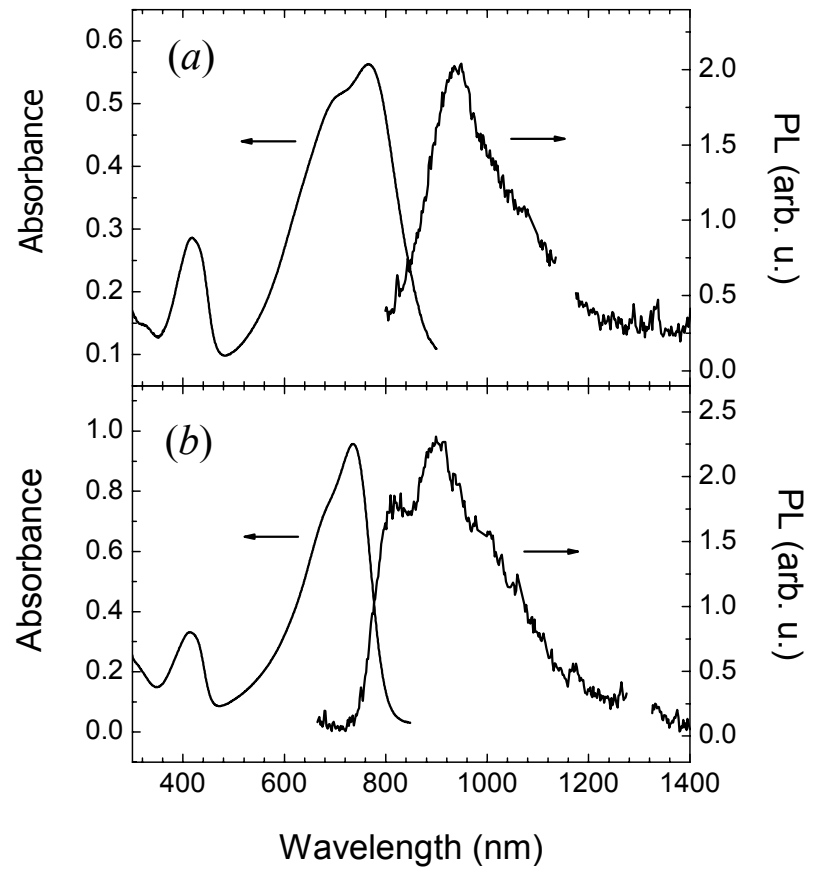

Fig. 2. Absorption and photoluminescence (PL) spectra of PCPDTBT: $(a)$ solid film and $(b)$ in chlorobenzene solution. PL spectra were obtained with an excitation wavelength of $\lambda=750 \mathrm{~nm}$.

A dual-beam femtosecond spectrometer that comprised of a visible pump generated by the second harmonic of the OPA and a white light continuum probe was employed for ultrafast transient absorption (TA) measurement. The TA spectra were measured by probing the decay at each wavelength using constant pump fluence. A Ge detector was used for detecting the TA signals in the near IR. The TA decay time constants were deduced by a least-squares deconvolution fitting assuming a gaussian time-profile of the pump with FWHM of $120 \mathrm{fs}$.

The film morphology was studied by a JEM-1230 transmission electron microscope operated at $80 \mathrm{kV}$. 


\section{RESULTS AND DISCUSSION}

\subsection{Absorption and Photoluminescence}

Fig. 2 shows the absorption and PL spectra of PCPDTBT. In the solid state (Fig. 1a) PCPDTBT exhibits optical absorption over the wide spectral range from the ultraviolet to $\lambda \sim 900 \mathrm{~nm}$, with peak at $\lambda \sim 768 \mathrm{~nm}$. From the onset of the absorbance, the optical gap of PCPDTBT is estimated to be $\mathrm{E}_{\mathrm{g}} \sim 1.46 \mathrm{eV}(\lambda=850 \mathrm{~nm})$. In ortho-dichlorobenzene solution (Fig. $2 b$ ), the absorption peak is located at $\lambda \sim 730 \mathrm{~nm}$, considerably red-shifted compared to the solid film; this may be indicative of interchain interactions as well as planarization/extension of the conjugated segments occurring in the solid state. The extension of the optical absorption of PCPDTBT in the near-infrared (NIR) considerably reduces the spectral mismatch with solar emission typical of higher bandgap polymers such as P3HT $\left(\mathrm{E}_{\mathrm{g}} \sim 1.8 \mathrm{eV}\right)$. This translates in a potential increase in solar cell efficiency of approximately a factor of two compared to P3HT-based devices.

The PL spectra of PCPDTBT film and solution are also shown in Fig. $2 a$ and $2 b$, respectively, with emission peaks at $\lambda \sim 950 \mathrm{~nm}$ and $\lambda \sim 900 \mathrm{~nm}$. The PL intensity is quite low compared to other emissive polymers (e.g. the PPVs), a result that may indicate a dipole-forbidden ${ }^{1} \mathrm{~A}_{\mathrm{g}}$ state as lowest excited state, similar to that observed in P3HT. ${ }^{20}$ Incorporating PCBM (50\% by weight) into the PCPDTBT resulted in total quenching the PL intensity (by a factor greater than 50 ). Energy transfer could be ruled out because of the bandgap of PCBM $(\sim 1.76 \mathrm{eV})$ is larger than that of PCPDTBT $(\sim 1.46$ $\mathrm{eV}$ ). Thus the total quenching of the PL is indicative of efficient electron transfer and charge separation in the PCPDTBT:PCBM composite.

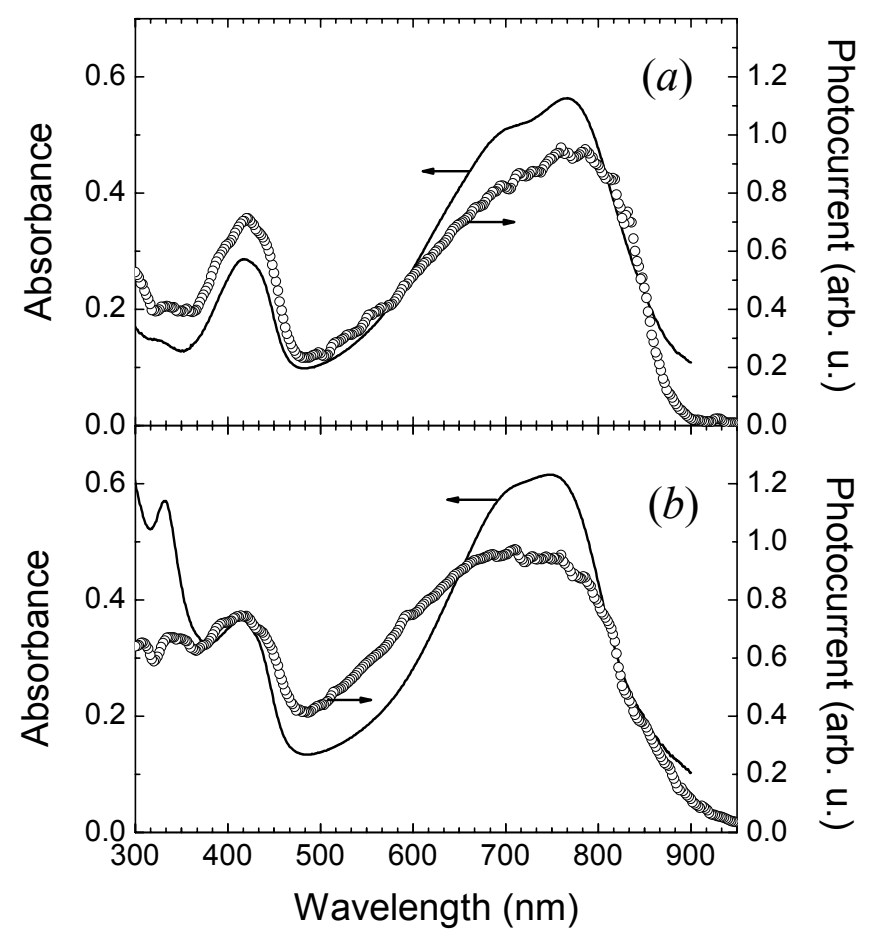

Fig. 3. Steady-state photocurrent and absorption spectra of: $(a)$ pristine PCPDTBT and $(b)$ PCPDTBT:PCBM (1:1) composite.

\subsection{Photoconductivity}

Fig. 3 shows the normalized steady-state PC spectra of pristine PCPDTBT (a) and of a PCPDTBT:PCBM blend $(b)$, compared to their optical absorption. Addition of PCBM does not significantly alter the absorption properties of 
PCPDTBT: the new spectral feature that appears in the spectrum of the blend at about $350 \mathrm{~nm}$ originates from PCBM. In both, pristine PCPDTBT and PCPDTBT:PCBM composite, the onset of the photocurrent coincides with the onset of the optical absorption. The photoconductivity is strongly sensitized in the PCPDTBT:PCBM composite and the photocurrent increases by about two orders of magnitude when PCBM is added to PCPDTBT. ${ }^{21}$ This is typical of polymer:fullerene composites where electron transfer from the polymer to the fullerene acceptor occurs, thus increasing the yield of charge carrier photogeneration and preventing geminate carrier recombination. ${ }^{22}$ The overall photoresponsivity of PCPDTBT:PCBM is comparable to that of the P3HT:PCBM model system (Fig. 4).

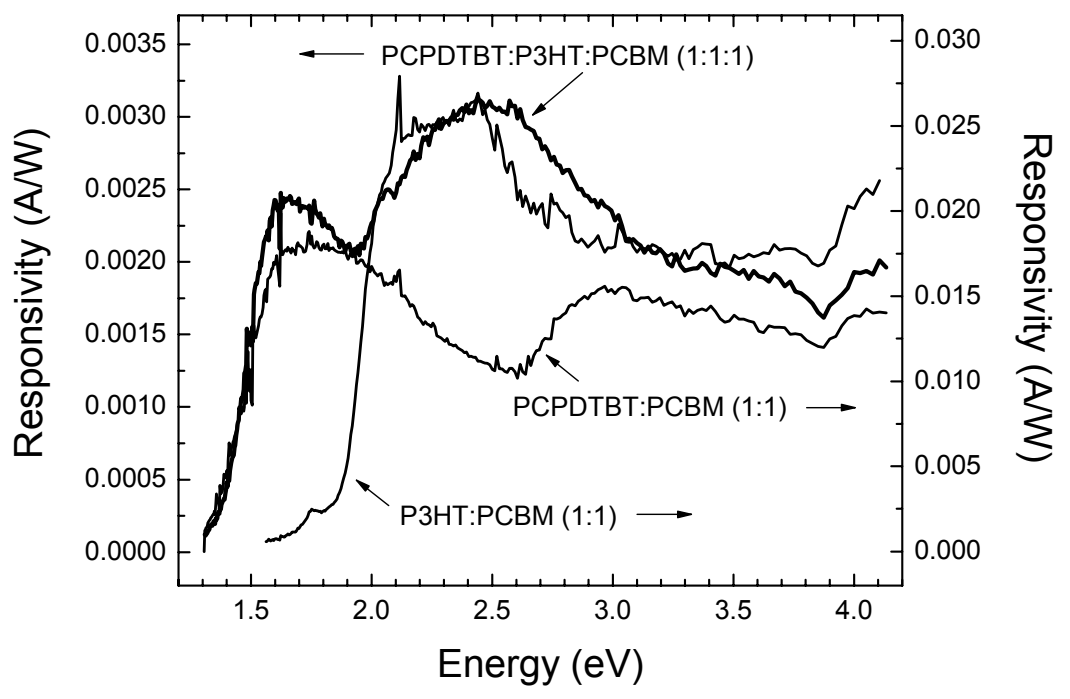

Fig. 4. Photoresponsivity of PCPDTBT:P3HT:PCBM (1:1:1), PCPDTBT:PCBM (1:1) and P3HT:PCBM (1:1) blends.

Low-bandgap polymers could be possibly used in "unconventional" photovoltaic architectures, such as tandem solar cells, where the superposition of two active layers with non-overlapping optical absorption allows improving the collection of the available solar power, or alternatively in multi-component bulk heterojunctions. In order to explore this latter possibility, we have also measured the steady-state photoconductivity spectra of the PCPDTBT:P3HT:PCBM composite, as shown in Fig. 4. Interestingly, we find that the photoconductive response of the multi-composite is considerably high over a broad spectral range, resembling the superposition of the photoconductivity of the single constituents.

In order to further investigate the effect of charge transfer on the carrier lifetime in PCPDTBT:PCBM blends and compare them to those of P3HT:PCBM, we have carried out fast ( $\mathrm{t}>100 \mathrm{ps})$ transient photocurrent measurements. Charge carrier photogeneration and recombination in the pristine polymers (both in P3HT and PCPDTBT) occur on a time scale faster than the temporal resolution of our measuring system $(\sim 100 \mathrm{ps})$, and therefore could not be resolved. The peak intensity of the resolution-limited transient photocurrent traces of the pristine polymers, however, increases significantly ( $\sim 10$ fold $)$ after addition of PCBM. Fig. 5 shows the normalized transient PC waveforms obtained from PCPDTBT:PCBM $(a)$ and P3HT:PCBM $(b)$ bulk heterojunctions. The photocurrent decay profiles in the two systems are almost identical, with a charge carrier lifetime deduced from the transient PC measurements of $\tau \sim 1.5$ ns. The appearance of a longer-lived photocurrent "tail" after addition of PCBM is typical of polymer:fullerene blends where ultrafast charge-transfer and carrier separation result in increased carrier lifetime. ${ }^{22}$ 


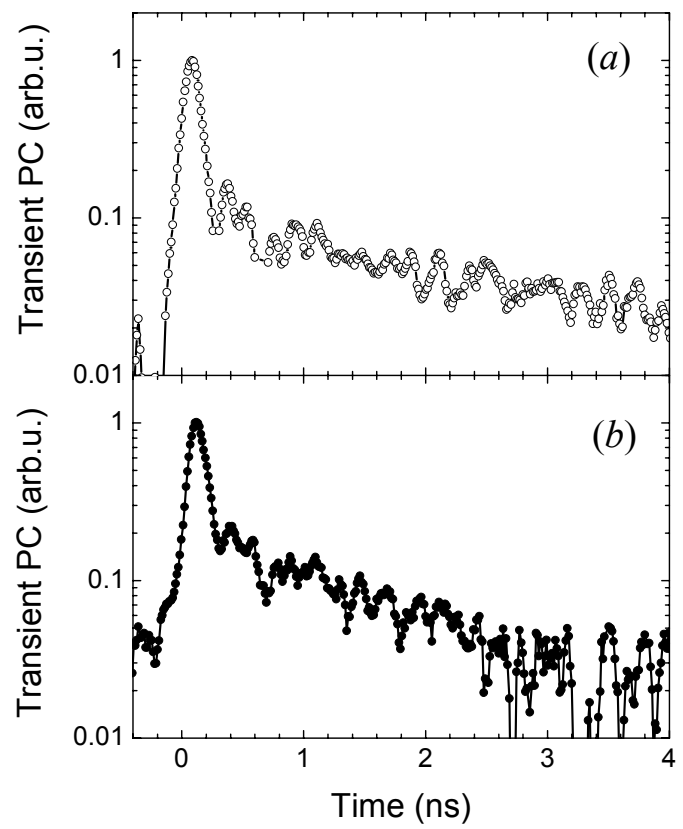

Fig. 5. Normalized transient photocurrent (PC) decay of: (a) PCPDTBT:PCBM (1:1) and (b) P3HT:PCBM (1:1) blends.

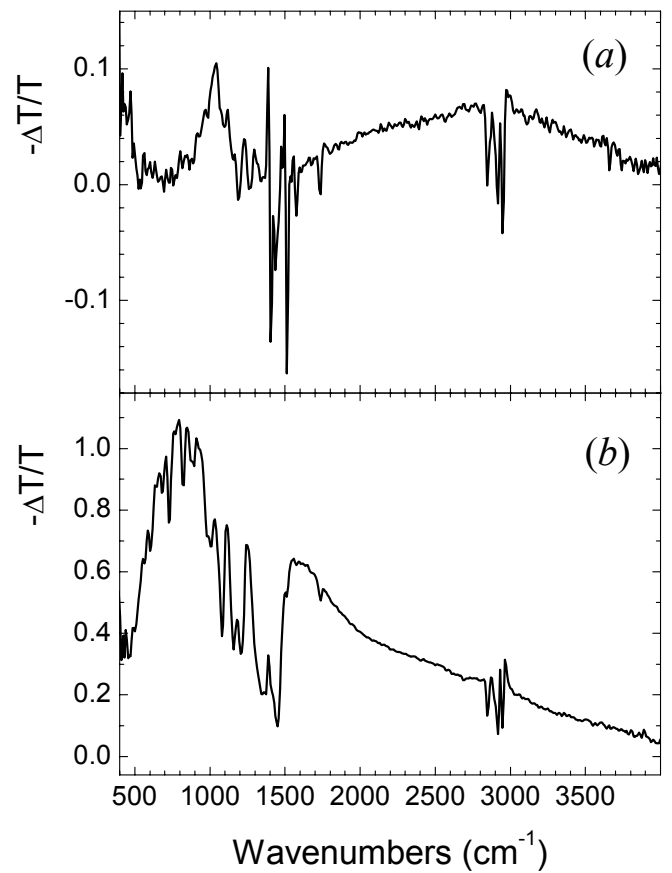

Fig. 6. Steady-state infrared photoinduced absorption (PIA) spectra of: (a) PCPDTBT:PCBM (1:1) and (b) P3HT:PCBM (1:1) composites, as measured at $\mathrm{T}=90 \mathrm{~K}$. 


\subsection{Photoinduced Absorption}

A typical spectroscopic signature of polaronic states in conjugated polymers is given by the appearance of new bands in the IR absorption spectrum. Two photoinduced absorption bands are usually observed in the NIR and medium-infrared (MIR) regions, corresponding to the dipole-allowed optical transitions to and from polaronic states (usually referred as $\mathrm{P}_{1}$ and $\mathrm{P}_{2}$ ), together with the appearance of infra-red active vibrational modes on the far side of the IR spectrum. ${ }^{23}$ Fig. 6 shows the infrared steady-state photoinduced absorption spectra of PCPDTBT:PCBM $(a)$ and P3HT:PCBM $(b)$. In P3HT:PCBM, the overlap of the IRAV modes with the $P_{1}$ band (peaked around $1200 \mathrm{~cm}^{-1}$ ) results in Fano antiresonances, as was described in Ref. ${ }^{24}$. In PCPDTBT:PCBM the $\mathrm{P}_{1}$ photoinduced absorption peak is significantly blue shifted (the broad PIA band centered around $2700 \mathrm{~cm}^{-1}$ ), indicative of a higher polaron relaxation energy compared to P3HT $\left(\mathrm{E}_{\text {rel }} \sim 0.3 \mathrm{eV}\right)$. Consequently, IRAV modes are clearly identifiable around $1000 \mathrm{~cm}^{-1}$, confirming the efficient photogeneration of charged species.

Fig. 7 shows the visible-NIR transient absorption (TA) spectra measured in pristine PCPDTBT $(a)$ and PCPDTBT:PCBM $(b)$. The PCPDTBT TA spectrum exhibits a photo-bleaching band centered at $\sim 800 \mathrm{~nm}$ and two PIA bands at 525 and $\sim 1400 \mathrm{~nm}$ and a decay time constant of the order of $100 \mathrm{ps}$. The TA spectrum of PCPDTBT:PCBM is dramatically different from that of PCPDTBT, even at very short time after photoexcitation $(t=1 \mathrm{ps})$. Note in particular that an additional PIA band appears at $950 \mathrm{~nm}$. This PIA band is relatively long-lived and thus ascribed to a charge transfer state subsequent to the electron transfer from PCPDTBT to PCBM. The PIA band from PCPDTBT:PCBM at $\sim 1400 \mathrm{~nm}$ is much longer-lived than that found for PCPDTBT at the same wavelength; this may indicate that the two have a different origin. The overall decay dynamics in PCPDTBT:PCBM is much slower than in PCPDTBT, compatible with the long charge carrier recombination time deduced by transient photocurrent measurements.

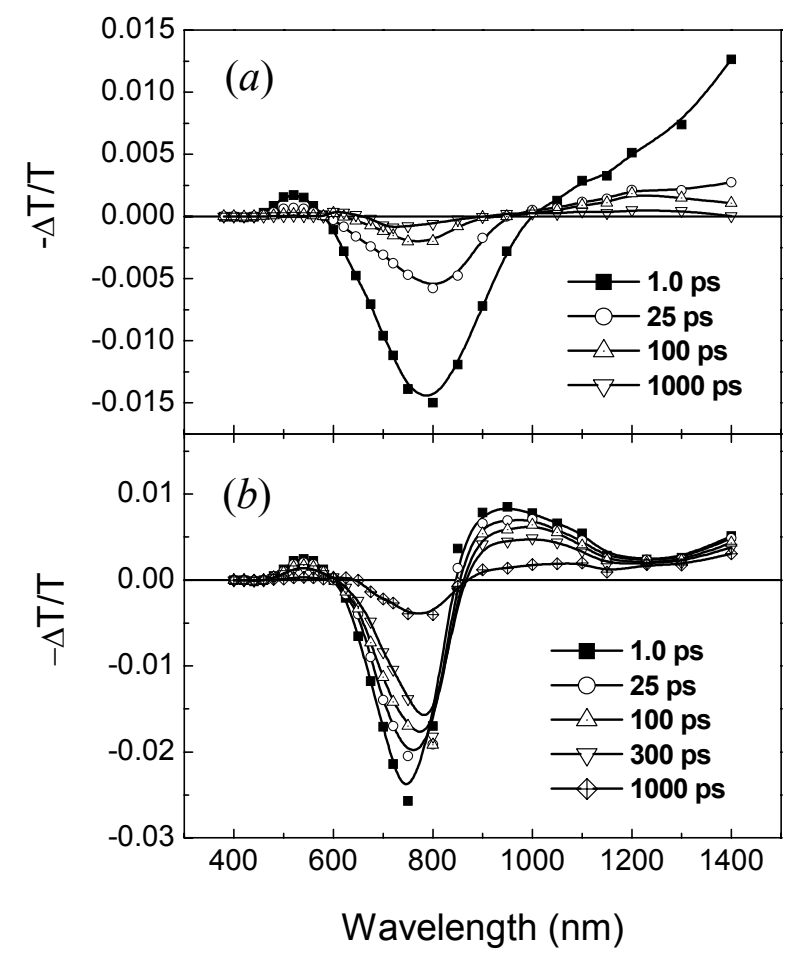

Fig. 7. Transient absorption spectra of: (a) pristine PCPDTBT and (b) PCPDTBT:PCBM (1:1) blend, as measured for several delay times and pump wavelength of $\lambda=720 \mathrm{~nm}$. The corresponding delay times are indicated in the figure.

A more complete picture of the electron transfer process in the PCPDTBT:PCBM composite is obtained from measurements of the TA decays at the ultrafast time scale (Fig. 8). The PIA signal probed at $525 \mathrm{~nm}$ rises within the 
experimental temporal resolution and exhibits an ultrafast decay characterized by a time constant of 585 fs. The PIA signal probed at $1000 \mathrm{~nm}$ shows a rise time similar to the decay time at $525 \mathrm{~nm}$; fitting the data to an exponential term yields a rise time constant of 505 fs. The PIA band centered at $525 \mathrm{~nm}$ in PCPDTBT:PCBM (that matches the one in PCPDTBT), is therefore ascribed to the singlet state absorption of the polymer, while the PIA band at $950 \mathrm{~nm}$ (that appears only in the PCPDTBT:PCBM composite) to the formation of a charge transferred state. The nearly identical values of the decay time probed at $525 \mathrm{~nm}$ and the rise time probed at $1000 \mathrm{~nm}$ implies that these two bands characterize the electron transfer process involving the dissociation of the singlet exciton in the polymer and build-up of the charge transferred state in PCPDTBT:PCBM. From the average of the two time constants (i.e. 505 and 585 fs), we deduce an electron transfer time from PCPDTBT to PCBM of $550 \mathrm{fs}$. The transient absorption decay profiles of pristine PCPDTBT probed in the photobleaching region reveals a fast decay component with lifetime of $\tau_{\mathrm{s}} \sim 60 \mathrm{ps}$ that can be attributed to the decay of the singlet state. ${ }^{25}$ Since the electron transfer time $(\sim 550 \mathrm{ps})$ is shorter by two orders of magnitude than the singlet state lifetime ( $\sim 60 \mathrm{ps})$, the quantum efficiency for electron transfer in PCPDTBT:PCBM should approach $100 \%$.

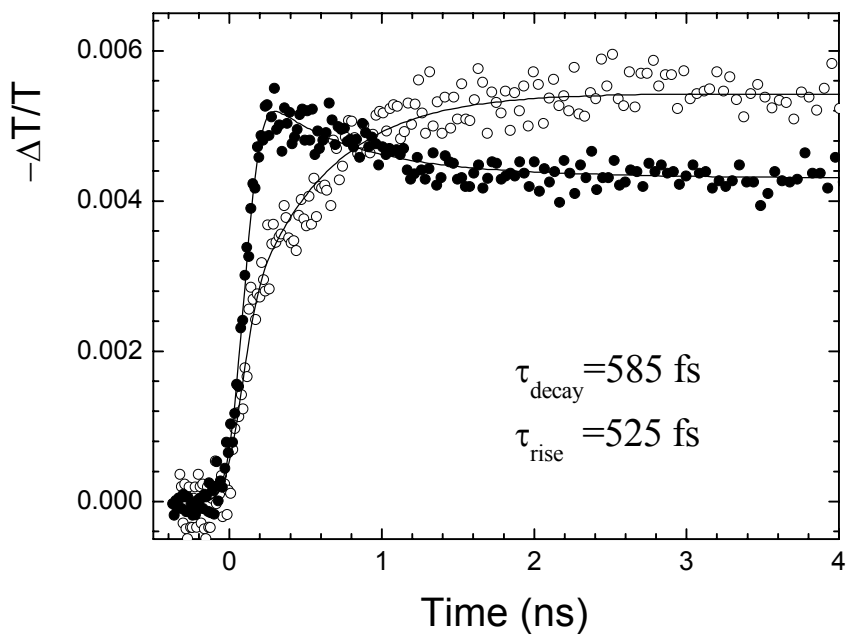

Fig. 8. Ultrafast transient absorption profiles of PCPDTBT:PCBM (1:1), measured with pump at $\lambda=720 \mathrm{~nm}$ and probe at $\lambda=525 \mathrm{~nm}$ (solid circles) and $\lambda=1000 \mathrm{~nm}$ (open circles). To facilitate comparison, the data obtained at $\lambda=1000 \mathrm{~nm}$ have been multiplied by a factor of 3.75 . The solid curves are exponential fits to the data at $\lambda=525 \mathrm{~nm}$ and $\lambda=1000 \mathrm{~nm}$, for which a decay time of $585 \mathrm{fs}$ and a rise time of $525 \mathrm{fs}$ are obtained, respectively.

\subsection{Morphology}

The results of extended NIR absorption, PC sensitization and highly efficient electron transfer in PCPDTBT:PCBM composites are encouraging toward the realization of high efficiency photovoltaics. So far, power conversion efficiencies as high as 3.3\% have been obtained in PCPDTBT:PCBM (1:3.3) bulk heterojunction solar cells, limited by a rather low fill-factor of the devices $(\mathrm{FF}<50 \%){ }^{26}$ It has been previously demonstrated that the polymer/fullerene ratio and annealing conditions significantly affect the morphology of the active layer and consequently the power conversion efficiency of BHJ photovoltaic cells. ${ }^{3,45}$ Fig. 9 shows the TEM micrographs of PCPDTBT:PCBM and P3HT:PCBM interpenetrating networks before and after annealing at $150{ }^{\circ} \mathrm{C}$ for 30 minutes. As can be seen from the pictures, the morphology of the PCPDTBT:PCBM $(1: 1)$ composite $(a, b)$ is very similar to that of the P3HT:PCBM $(1: 1)$ blend $(e, f)$. In these composites thermal annealing improves the morphology of the donor-acceptor interpenetrating network (the donor-acceptor domains are better defined), thus resulting in larger interfacial area for efficient charge generation. On the other hand, the morphology of the PCPDTBT:PCBM (1:4) interpenetrating network $(c)$ is poorer and degrades even further after thermal annealing $(d)$ due to the formation of large PCBM aggregates. The low fill factor achieved in PCPDTBT-based photovoltaics so far may therefore be a consequence of the poor morphology of the interpenetrating 
network at the high concentration of PCBM required for optimal efficiency: a reduced packing of the polymer chains in PCPDTBT as compared to the lamellar structure of $\mathrm{P}_{3} \mathrm{HT}^{3,27}$ may be critical for the charge transport and extraction in photovoltaic cells, thus limiting the conversion efficiency.
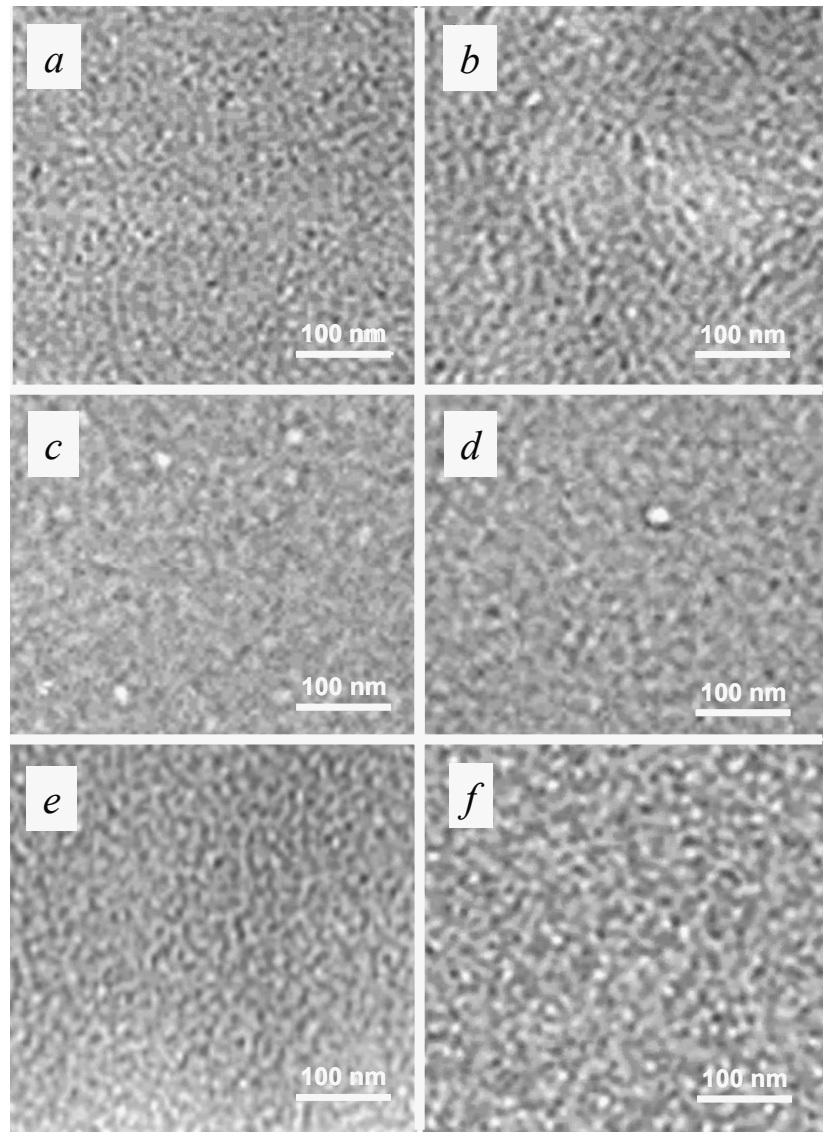

Fig. 9. TEM images of PCPDTBT:PCBM (1:1) before $(a)$ and after $(b)$ annealing, PCPDTBT:PCBM $(1: 4)$ before $(c)$ and after $(d)$ annealing, and P3HT:PCBM $(1: 1)$ before $(e)$ and after $(f)$ annealing. Thermal annealing was performed in a vacuum oven at $150{ }^{\circ} \mathrm{C}$ for 30 minutes.

\section{SUMMARY AND CONCLUSION}

We have studied the charge carrier photogeneration and transport properties of the low-bandgap conjugated polymer PCPDTBT and of the PCPDTBT:PCBM bulk heterojunction material by a combination of several experimental techniques. Since the optical absorption and the photoconductive response of PCPDTBT extends from the UV to the NIR (up to $\lambda \sim 900 \mathrm{~nm}$ ), this system allows improved light harvesting in polymer-based solar cells. The PCPDTBT:PCBM bulk heterojunction material shows ultrafast electron transfer reaction with quantum efficiency approaching unity that results in long-lived charge-separated states. The picture of the photophysics of PCPDTBT:PCBM emerging from our studies is in every respect similar to that of P3HT:PCBM, to date the best system for polymer-based photovoltaics. Thus, PCPDTBT could be used as the active component in photovoltaic devices such as PCPDTBT:PCBM and PCPDTBT:P3HT:PCBM bulk heterojunctions, or tandem solar cells. Comprehensive studies of the performance of such devices are underway with the goal of optimizing the photovoltaic efficiency. 


\section{ACKNOWLEDGMENTS}

Research at UCSB was supported by Konarka Technologies, Inc. The assistance of Dr. Alexander Mikhailovsky in photoluminescence measurements is gratefully acknowledged. We also thank Mr. Wanli Ma and Dr. Jin Young Kim for the useful discussions.

\section{REFERENCES}

1. Basic Research Needs for Solar Energy Utilization, Report on the Basic Energy Sciences Workshop on Solar Energy Utilization, April 18-21 2005, available on the web at http://www.sc.doe.gov/bes/reports/files/SEU_rpt.pdf.

2. Martin A. Green, Third Generation Photovoltaics: Ultra-high Conversion Efficiency at Low Cost, Prog. Photovolt.: Res. Appl. 9, 123-135 (2001); Martin A. Green, Third Generation Photovoltaics: Advanced Solar Energy Conversion. Springer-Verlag: Berlin, Germany (2004).

3. W. Ma, W., C. Yang, X. Gong, K. Lee, and A. J. Heeger, Thermally stable, efficient polymer solar cells with nanoscale control of the interpenetrating netwok morphology, Adv. Funct. Mat. 15, 1617 (2005).

4. G. Li et al., High-efficiency solution processable polymer photovoltaic cells by self-organization of polymer blends, Nat. Mater. 4, 864 (2005).

5. M. Reyes-Reyes, K. Kim, and D. L. Carrol, High-efficiency photovoltaic devices based on annealed poly(3hexylthiophene) and 1-(3-methoxycarbonyl)-propyl-1-phenyl-(6,6) $C_{61}$ blends, Appl. Phys. Lett. 87, 083506 (2005).

6. L. J. A. Koster et al., Ultimate efficiency of polymer/fullerene bulk heterojunction solar cells, Appl. Phys. Lett. 88, 093511 (2006).

7. S. R. Forrest, The Limits to Organic Photovoltaic Cell Efficiency, MRS Bulletin 30, 28 (2005).

8. K. M. Coakley and M. D. McGehee, Conjugated Polymer Photovoltaic Cells, Chem. Mater. 16, 4533 (2004).

9. G. Li, V. Shrotriya, Y. Yao, Y. Yang, Investigation of annealing effects and film thickness dependence of polymer solar cells based on poly(3-hexylthiophene), J. Appl. Phys. 98, 43704 (2005).

10. F. Padinger, R. S. Rittberger, N. S. Sariciftci, Effects of Postproduction Treatment on Plastic Solar Cells, Adv. Funct. Mater. 13, 85 (2003).

11. J. Roncali, Synthetic Principles for Bandgap Control in Linear $\pi$-Conjugated Systems. Chem. Rev. 971, 73 (1997).

12. A. Dhanabalan et al., Synthesis and characterization of a low bandgap conjugated polymer for bulk heterojunction photovoltaic cells, Adv. Funct. Mat. 11, 255 (2001).

13. J. K. J. van Duren, A. Dhanabalan, P. A. van Hal, R. A. J. Janssen, Low-bandgap polymer photovoltaic cells, Synth. Met. 121, 1587 (2001).

14. C. J. Brabec et al., A low-bandgap semiconducting polymer for photovoltaic devices and infrared emitting diodes, Adv. Funct. Mater. 12, 709 (2002).

15. C. Winder et al., Sensitization of low bandgap polymer bulk heterojunction solar cells, Thin Solid Films 403, 373 (2002).

16. M. Svensson et al., High-Performance Polymer Solar Cells of an Alternating Polyfluorene Copolymer and a Fullerene Derivative, Adv. Mater. 15, 988 (2003).

17. K. G. Jespersen et al., The electronic states of polyfluorene copolymers with alternating donor-acceptor units, J. Chem. Phys. 121, 12613 (2004).

18. Z. Zhu, R. Kingsborough, R. Gaudiana and D. Waller, to be published.

19. D.H. Auston, Impulse-Response of Photoconductors in Transmission-Lines, IEEE Journal of Quantum Electronics 19, 639 (1983).

20. R. Österbacka et al., Two-Dimensional Electronic Excitations in Self-Assembled Conjugated Polymer Nanocrystals, Science 287, 839 (2000).

21. C. Soci et al., Photoconductivity of a Novel Low-Bandgap Conjugated Polymer, Adv. Funct. Mat., in press.

22. C. H. Lee et al., Sensitization of the photoconductivity of conducting polymers by $C_{60}$ : Photoinduced electron transfer, Phys. Rev. B 48, 15425 (1993).

23. B. Horovitz, Infrared activity of Peierls systems and application to polyacetylene, Solid State Comm. 41, 729 (1982).

24. R. Österbacka et al., Photoinduced quantum interference antiresonances in pi-conjugated polymers, Phys. Rev. Lett. 88, 226401 (2002). 
25. In-Wook Hwang et al., Ultrafast Electron Transfer from a Novel Low Bandgap Polymer to Fullerene, to be published.

26. Jin Young Kim, unpublished results.

27. C.Y. Yang, C. Soci, D. Moses, A.J. Heeger, Aligned rrP3HT film: Structural order and transport properties, Synth. Met. 155, 639 (2005). 